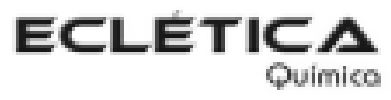

www.scielo.br/eq

Volume 29, número 1, 2004

\title{
Influência da taxa de dose na degradação do herbicida ácido diclorofenóxiacético $(2,4-d)$ por meio da radiação gama do cobalto-60
}

\author{
S. X. de Campos ${ }^{1}$, S. M. Sanches ${ }^{1}$, S. Z. Falone ${ }^{1}$, E. M. Vieira ${ }^{1 *}$ \\ ${ }^{1}$ Instituto de Química de São Carlos - Universidade de São Paulo - CP 780 - CEP 13566-590 -São Carlos - SP.
}

Resumo: Métodos convencionais de descontaminação ambiental, algumas vezes apenas transferem esses resíduos de um lugar para outro. Esse estudo tem como objetivo checar a influência de diferentes taxas de doses de radiação gama do cobalto-60 na degradação do herbicida 2,4-D, em água e metanol. Os resultados mostraram que o 2,4-D, em água, foi totalmente degradado em uma dose de $30 \mathrm{kGy}$, utilizando-se taxa de dose de $2,7 \mathrm{kGy} \mathrm{h}^{-1}$ e $20 \mathrm{kGy}$ com uma taxa de dose de 5- $60 \mathrm{kGy} \mathrm{h}^{-1}$. Para o metanol, a total degradação do 2,4-D ocorreu com uma dose de $150 \mathrm{kGy}$ e taxa de dose de 2,7 kGy h-1 e $100 \mathrm{kGy}$ utilizando-se taxa de dose de $5-60 \mathrm{kGy} \mathrm{h}^{-1}$. Assim conclui-se que a degradação do herbicida 2,4-D é dependente da dose e da taxa de dose de radiação.O valor radiolítico da degradação do 2,4-D foi calculado.

Palavras-chave: 2,4-D, degradação, taxa de dose, radiação gama

\section{Introdução}

Como uma conseqüência do rápido crescimento industrial e agrícola, o ambiente tem-se tornado cada vez mais contaminado com poluentes. Em particular ,nos sistemas aquáticos, o intensivo uso de fertilizantes e pesticidas e a geração de um vasto número de resíduos têm contribuído para a degradação da qualidade da água.

O herbicida ácido diclorofenóxiacético (2,4D) foi produzido durante o programa da guerra química e biológica no período da segunda Guerra Mundial (1939-1945), sendo também utilizado na guerra do Vietnã (1954-1975), fazendo parte, juntamente com o herbicida 2,4,5-T, de um composto conhecido como agente laranja, que era utilizado como desfolhante das florestas Vietnamitas [1]. Desde então o 2,4-D vem sendo utilizado no controle seletivo de ervas daninhas. Embora estudos farmacológicos demonstrem que ele não é acumulado no corpo humano, em 1982 a Organização
Mundial da Saúde (OMS) considerou o 2,4-D como moderadamente tóxico (classe II) e recomendou uma concentração máxima na água de abastecimento de 100 mg L-[1 2$]$.

Muitos pesquisadores, em diferentes países têm utilizado a radiação gama, como indutora da decomposição dos mais diversos poluentes ambientais, como por exemplo, no tratamento de efluentes domésticos, poluentes tóxicos em efluentes industriais, degradação de pesticidas e descontaminação de água e solo $[3,4,5,6,11,12$, 13,14,15,16,17].

Quando água ou metanol são irradiados com radiações ionizantes, como radiação gama, várias espécies são formadas. A primeira consequiência da radiólise gama da água é a formação de espécies excitadas, que por sua vez se decompõem em espécies reativas. Estas espécies, ao reagirem entre si e com outras moléculas presentes, podem formar outras espécies, tais como radicais, íons, elétrons aquosos, átomos de hidrogênio, produtos moleculares e produtos

*e-mail: eny@iqsc.sc.usp.br; Fax: (55 16) 273-9985 
gasosos [10] como apresentado a seguir, onde as espécies formadas prioritariamente são $\mathrm{OH} \cdot \mathrm{e} \mathrm{e}_{\text {aq. }}^{-}$

$$
\begin{aligned}
& \mathrm{H}_{2} \mathrm{O}+\gamma \rightarrow \mathrm{H}_{2} \mathrm{O}^{+}+\mathrm{e}_{\text {aq }}^{-} \\
& \mathrm{H}_{2} \mathrm{O}+\gamma \rightarrow \mathrm{H}_{2} \mathrm{O}^{\bullet} \\
& \mathrm{e}_{\text {aq }}^{-}+\mathrm{H}_{2} \mathrm{O} \rightarrow \mathrm{H}_{2} \mathrm{O}^{-} \\
& \mathrm{H}_{2} \mathrm{O}^{+}+\mathrm{H}_{2} \mathrm{O} \rightarrow \mathrm{H}_{3} \mathrm{O}^{+}+{ }^{\cdot} \mathrm{OH} \\
& \mathrm{H}_{2} \mathrm{O}^{-} \rightarrow \mathrm{OH}^{-}+\mathrm{H}^{\cdot} \\
& \mathrm{H}_{2} \mathrm{O}^{\bullet} \rightarrow\left[\mathrm{H}^{\bullet}+{ }^{\bullet} \mathrm{OH}\right] \rightarrow \mathrm{H}_{2} \mathrm{O}(6) \\
& \mathrm{H}_{2} \mathrm{O}^{-}+\mathrm{H}_{3} \mathrm{O}^{+} \rightarrow \mathrm{H}_{2} \mathrm{O}+\mathrm{H}^{\bullet} \\
& \mathrm{H}^{\bullet}+{ }^{\cdot} \mathrm{OH} \rightarrow \mathrm{H}_{2} \mathrm{O} \\
& \mathrm{H}^{\bullet}+\mathrm{H}^{\bullet} \rightarrow \mathrm{H}_{2} \\
& \cdot \mathrm{OH}+\cdot \mathrm{OH} \rightarrow \mathrm{H}_{2} \mathrm{O}_{2}
\end{aligned}
$$

Estas reações de recombinação ocorrem em um período de aproximadamente $10^{-9}$ segundos.

Segundo Spinks \& Woods[18], na radiólise gama do metanol líquido verifica-se a formação dos seguintes produtos primários:

$$
\begin{aligned}
\mathrm{CH}_{3} \mathrm{OH}+\gamma & \rightarrow \mathrm{e}_{\text {solv }}^{-}+{ }^{\bullet} \mathrm{CH}_{2} \mathrm{OH}+\mathrm{H}^{\bullet}(11) \\
& \rightarrow \mathrm{CH}_{3} \mathrm{OH}^{\bullet} \\
& \rightarrow \mathrm{CH}_{3}+{ }^{\bullet} \mathrm{OH} \\
& \rightarrow \mathrm{CH}_{3} \mathrm{OH}^{+}+\mathrm{e}_{\text {solv }}^{-} \\
& \rightarrow \mathrm{CH}_{3} \mathrm{O}^{\bullet}+\mathrm{H}^{+}
\end{aligned}
$$

Sob condições normais de radiólise, estes produtos podem participar de reações formando:

$$
\begin{aligned}
& \mathrm{CH}_{3} \mathrm{OH}^{+}+\mathrm{CH}_{3} \mathrm{OH} \rightarrow \mathrm{CH}_{3} \mathrm{OH}_{2}^{+}+{ }^{\circ} \mathrm{CH}_{2} \mathrm{OH}\left(\mathrm{ou} \mathrm{CH}_{3} \mathrm{O}^{\circ}\right)(16) \\
& \mathrm{CH}_{3} \mathrm{O}^{\bullet}+\mathrm{CH}_{3} \mathrm{OH} \rightarrow \mathrm{CH}_{3} \mathrm{OH}+{ }^{\cdot} \mathrm{CH}_{2} \mathrm{OH} \\
& \mathrm{e}_{\text {solv }}^{-}+\mathrm{CH}_{3} \mathrm{OH} \rightarrow \mathrm{CH}_{3} \mathrm{O}^{-}+\mathrm{H}^{*} \\
& \rightarrow{ }^{\circ} \mathrm{CH}_{3}+\mathrm{OH} \\
& \mathrm{CH}_{3} \mathrm{OH}^{+}+\mathrm{H}^{\cdot} \rightarrow \mathrm{CH}_{3} \mathrm{OH}_{2}^{+} \\
& \mathrm{CH}_{3} \mathrm{OH}_{2}^{+}+\mathrm{CH}_{3} \mathrm{O} \rightarrow 2 \mathrm{CH}_{3} \mathrm{OH} \\
& 2 \cdot \mathrm{CH}_{2} \mathrm{OH} \rightarrow \text { glicol } \\
& \cdot \mathrm{CH}_{3}+\mathrm{H}^{\cdot} \rightarrow \mathrm{CH}_{4} \\
& \cdot \mathrm{CH}_{3}+\mathrm{CH}_{3} \mathrm{OH} \rightarrow \mathrm{CH}_{4}+{ }^{\cdot} \mathrm{CH}_{2} \mathrm{OH} \\
& 2 \mathrm{H}^{\bullet} \rightarrow \mathrm{H}_{2} \\
& 2 \mathrm{CH}_{3} \mathrm{O}^{\bullet} \rightarrow \mathrm{CH}_{3} \mathrm{OH}+\mathrm{HCHO} \\
& \mathrm{H}^{\bullet}+\mathrm{CH}_{3} \mathrm{O}^{\bullet} \rightarrow \mathrm{H}_{2}+\mathrm{HCHO} \\
& \mathrm{CH}_{3} \mathrm{O}^{\bullet}+\mathrm{HCHO} \rightarrow \mathrm{CH}_{3} \mathrm{OH}+{ }^{\circ} \mathrm{CHO} \\
& \cdot \mathrm{CHO}+\mathrm{CH}_{3} \mathrm{O}^{\bullet} \rightarrow \mathrm{CH}_{3} \mathrm{OH}+\mathrm{CO}
\end{aligned}
$$

A reação dos radicais livres com metanol produz:

$$
\begin{array}{ll}
\mathrm{H}^{\bullet}+\mathrm{CH}_{3} \mathrm{OH} & \rightarrow{ }^{\circ} \mathrm{CH}_{2} \mathrm{OH}+\mathrm{H} \text { (30) } \\
{ }^{\cdot} \mathrm{CH}_{3}+\mathrm{CH}_{3} \mathrm{OH} & \rightarrow{ }^{\circ} \mathrm{CH}_{2} \mathrm{OH}+\mathrm{CH}_{4} \\
2^{\cdot} \mathrm{CH}_{2} \mathrm{OH} & \rightarrow \mathrm{C}_{2} \mathrm{H}_{6} \mathrm{O}_{2}
\end{array}
$$

Assim, as principais espécies formadas na radiólise gama do metanol são: $\mathrm{H}^{\bullet},{ }^{\bullet} \mathrm{CH}_{2} \mathrm{OH}, \mathrm{H}_{2}$, $\mathrm{CH}_{4} \cdot{ }^{\circ} \mathrm{CH}_{3} \mathrm{CO}, \mathrm{HCHO}$ e $\mathrm{C}_{2} \mathrm{H}_{6} \mathrm{O}$ [18].

Neste trabalho buscou-se estudar diferentes taxas de dose de radiação (dose de radiação absorvida por um dado sistema em um determinado tempo e expressa em $\mathrm{kGy} \mathrm{h}^{-1}$ ), para a degradação do 2,4-D em água e metanol e também calcular os valores radiolíticos da degradação

\section{Procedimento Experimental}

\section{Irradiação das amostras}

As soluções do herbicida 2,4-D, em água e metanol, foram colocadas em tubos de vidro de borosilicato com volume de $5 \mathrm{~mL}$. Os tubos foram selados e enviados para serem irradiados à doses que variaram de 0 a $150 \mathrm{kGy}$.

As amostras foram irradiadas utilizando um irradiador JS 7500, fabricado pela empresa Nordion do Canadá, na Empresa Brasileira de Radiações, EMBRARAD em Cotia, São Paulo. As soluções foram irradiadas em dois diferentes sistemas no mesmo irradiador, com a finalidade de obter diferentes taxas de dose $\left(\right.$ dose tempo $\left.{ }^{-1}\right)$.

Em um dos sistemas as soluções foram acondicionadas dentro de caixas de alumínio com $90 \mathrm{~cm}$ de altura, $60 \mathrm{~cm}$ de comprimento e $50 \mathrm{~cm}$ de profundidade. Estas caixas foram colocadas na esteira de entrada para serem levadas à câmara de irradiação, onde percorreram um trajeto de aproximadamente 29 $\mathrm{m}$ em torno da fonte de cobalto-60 e voltaram pela esteira de saída. Este trajeto em torno da fonte de cobalto-60 é composto de 48 posições diferentes, sendo 24 no plano inferior de irradiação (chamado de bandeja inferior) e 24 no plano superior de irradiação (chamado de bandeja superior), a dose recebida pelas amostras é diretamente proporcional ao tempo em que a caixa de alumínio permanece em cada uma destas posições. Este tempo é ajustado através do painel de controle, e é chamado de ciclo ou passo da máquina. Em todo trajeto percorrido pela caixa de 
alumínio dentro do irradiador, a mesma não sofre qualquer movimento de rotação, somente translação. Este sistema é conhecido como irradiação dinâmica e através dele as soluções foram irradiadas a uma taxa de dose que variou de 5 a $60 \mathrm{kGy} \mathrm{h}^{-1}$.

A outra forma de irradiação empregada foi por meio da utilização de um equipamento conhecido como canal experimental ("research loop"). O canal experimental permite a irradiação de amostras sem interferir no funcionamento normal do irradiador. Assim as soluções foram irradiadas em um sistema composto por uma caixa de alumínio com paredes de aproximadamente $2 \mathrm{~mm}$ de espessura e com capacidade para 20 litros. Essa caixa de alumínio foi suspensa por trilhos que permitiram o seu movimento até a sua posição de irradiação estática. Neste sistema a irradiação é realizada em apenas uma posição do equipamento. Por meio deste sistema obtém-se uma taxa de dose constante de 2,7 $\mathrm{kGy} \mathrm{h}^{-1}$. Durante todas as irradiações o irradiador apresentava uma atividade de $92 \mathrm{k}$ Curie ${ }^{60} \mathrm{Co}$.

\section{Reagentes e soluções}

O herbicida 2,4-D, massa molar $=221,04$, foi adquirido junto a Laboratórios Dr. Ehrenstorfer, Alemanha. A Figura 1 apresenta a estrutura química do 2,4-D.

As soluções do 2,4-D foram preparadas dissolvendo-se $25 \mathrm{mg}$ do herbicida em 1 litro de água ultrapura, Milli-O (18,2 MW. cm) e $25 \mathrm{mg}$ do herbicida em $1 \mathrm{~L}$ de metanol grade HPLC (MALLINCKRODT).

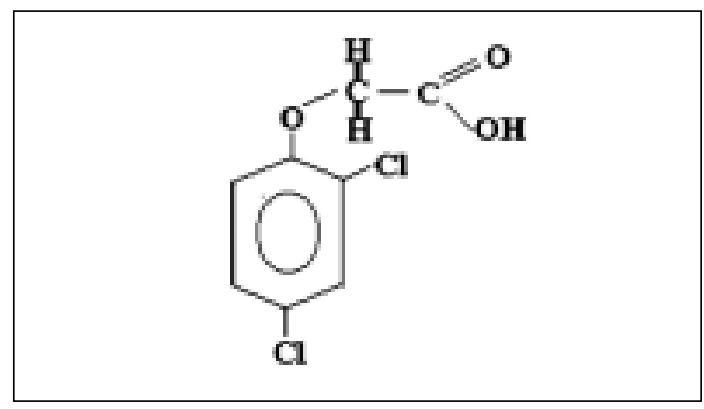

Figura 1 - Estrutura química do 2,4-D.

\section{Métodos analíticos}

O herbicida que permaneceu não degradado, em solução, nas diferentes doses de radiação, foi quantificado utilizando-se uma curva analítica obtida com padrões externos nas concentrações de $5,10,15,20$ e $25 \mathrm{mg} \mathrm{L}^{-1}$. Para a obtenção da curva analítica foi utilizado um cromatógrafo Shimadzu LC-9 com uma coluna RP - 18 HP (200 mm x 4,6 $\mathrm{mm} \times 10 \mathrm{~mm}$ ), detector UV-vis com comprimento de onda fixo em $280 \mathrm{~nm}$, fase móvel com $40 \%$ acetronitrila $+60 \%$ água com $2 \%$ de ácido acético, operando no modo isocrático, vazão de $1 \mathrm{~mL}$ min $^{-}$ 1 e volume injetado de $20 \mathrm{~mL}$. Estas condições foram selecionadas após estudos experimentais. Todas as amostras foram injetadas em triplicata.

Utilizando-se os resultados experimentais, calculou-se os valores radiolíticos $(\mathrm{G})^{*}$ que são apresentados na Tabela 1.

Tabela 1 - G-valores e razão de degradação para o herbicida 2,4-D irradiado em água e metanol, nas taxas de doses de 2,7 and 5-60 kGy h-1.

\begin{tabular}{c|c|c|c|c}
\hline $\begin{array}{c}\text { Taxas de } \\
\text { doses } \\
\mathrm{kGy} / \mathrm{h}\end{array}$ & $\begin{array}{c}\text { G- 2,4-D } \\
\text { em água }\end{array}$ & $\begin{array}{c}\text { G-2,4-D } \\
\text { em metanol }\end{array}$ & $\begin{array}{c}\text { Razão de degradação em } \\
\text { água (mol/ L kGy })\end{array}$ & $\begin{array}{c}\text { Razão de } \\
\text { degradação em metanol } \\
(\mathrm{mol} / \mathrm{L} \mathrm{kGy})\end{array}$ \\
\hline 2,7 & 0,81 & 0,10 & $8,44 \times 10^{-6}$ & $1,08 \times 10^{-6}$ \\
\hline $5-60$ & 1,04 & 0,14 & $1,08 \times 10^{-5}$ & $1,52 \times 10^{-6}$ \\
\hline
\end{tabular}

"O valor radiolítico (G) é o número de moléculas produzidas ou decompostas por $100 \mathrm{eV}$ de energia absorvida. Para conversão dentro do sistema internacional (SI) o valor radiolítico deve ser multiplicado por 0.10364, para obter os valores em $m \mathrm{~mol} \mathrm{~J}^{-1}$. Este valor é calculado a partir da regressão linear das concentrações do herbicida não degradado, em função das diferentes doses de radiação [7] . 


\section{Resultados e Discussão}

\section{Degradação radiolítica do 2,4-D}

As soluções do herbicida 2,4-D foram irradiadas em água nas doses de 1, 5, 10, 20 e 30 kGy e em metanol nas doses de $1,5,10,20,30,40,75$, $100,150 \mathrm{kGy}$, todos nas taxas de dose de 2,7 and $5-60 \mathrm{kGy} \mathrm{h}^{-1}$. As Figuras 2 e 3 mostram os resultados obtidos e a Figure 4 e 5 mostram alguns cromatogramas representativos do 2,4-D irradiado em água e em metanol nas duas taxas de dose.

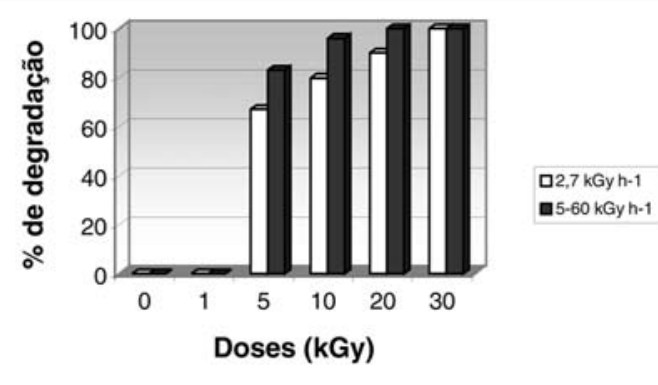

Figura 2 - Porcentagem de degradação do 2,4-D irradiado em água, em relação a dose de radiação e da taxa de dose.

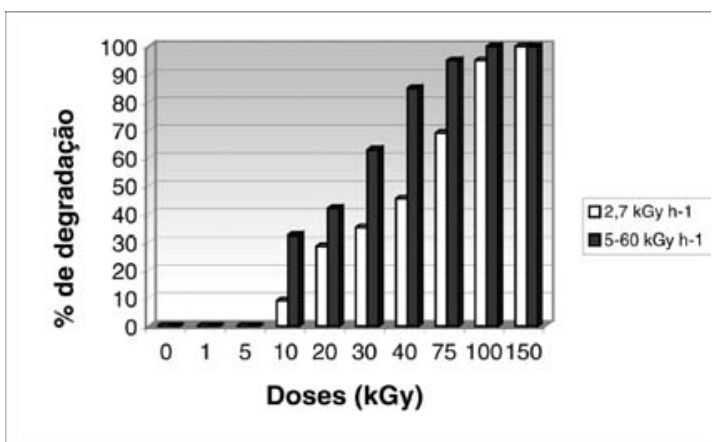

Figura 3 - Porcentagem de degradação do 2,4-D irradiado em metanol, em relação a dose de radiação e da taxa de dose.

Por meio dos resultados das Figuras 2 e 3 pode-se observar que a taxa de dose teve influência significativa na degradação do herbicida 2,4-D em água e em metanol. Enquanto que para taxa de dose maior (5-60 $\left.\mathrm{kGy} \mathrm{h}^{-1}\right)$ foi necessário uma dose de radiação de 20 kGy em água e 100 kGy em metanol para degradação total, para a taxa de dose menor 2,7 $\left(\mathrm{kGy} \mathrm{h}^{-1}\right)$ conseguiu-se a degradação total do herbicida depois de uma dose de radiação de 30 kGy em água e $150 \mathrm{kGy}$ em metanol.

Este comportamento é evidenciado nas Figuras 4 e 5 . Com mesma dose e taxas de doses diferentes o pico referente ao 2,4-D (tempo de retenção de 7,5 min.) mostrou uma maior área quando se utilizou a taxa de dose menor $\left(2,7 \mathrm{kGy} \mathrm{h}^{-1}\right)$.

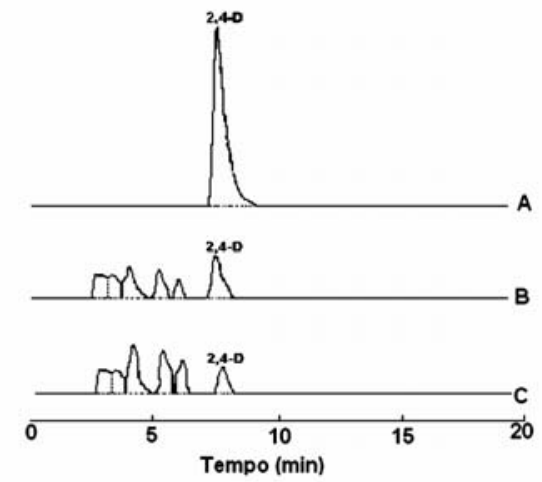

Figura 4 - Cromatogramas obtidos por HPLC para o 2,4-D irradiado em água: A) 2,4-D em água não irradiado; B) 2,4-D em água, irradiado com uma dose de $10 \mathrm{kGy}$ e taxa de dose de $2,7 \mathrm{kGy} \mathrm{h}^{-1}$; C) 2,4-D em água, irradiado com uma dose de 10 kGy e taxa de dose de 5- $60 \mathrm{kGy} \mathrm{h}^{-1}$.

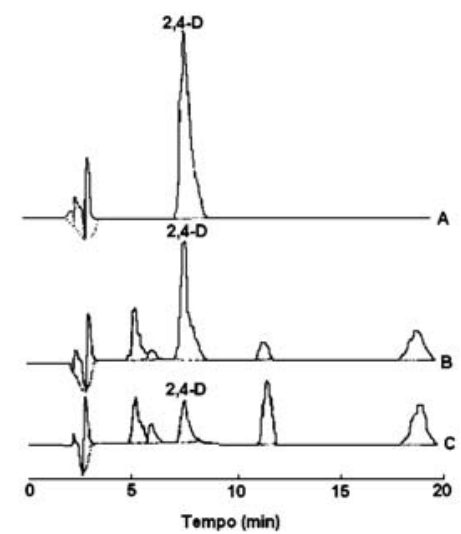

Figura 5 - Cromatogramas obtidos por HPLC para o 2,4-D irradiado em metanol: A) 2,4-D em metanol não irradiado; B) 2,4-D em metanol, irradiado com uma dose de $50 \mathrm{kGy}$ e taxa de dose de 2,7 $\mathrm{kGy} \mathrm{h}^{-1}$; C) 2,4-D em metanol, irradiado com uma dose de $50 \mathrm{kGy}$ e taxa de dose de $5-60 \mathrm{kGy} \mathrm{h}^{-1}$

Uma explicação para esse comportamento é a de que com a aplicação de uma dose maior em um período menor de tempo, as espécies mais 
reativas dos solventes $\left(\mathrm{OH}^{\bullet}, \mathrm{e}^{-}{ }_{\text {aq, }} \mathrm{H}^{\bullet}\right)$ formam-se em maior concentração e assim atacam mais efetivamente a molécula do herbicida, aumentando sua degradação [8].

Em presença de ar $\mathrm{H} \mathrm{e} \mathrm{e}_{(\text {aq) }}^{-}$são convertidos em radicais peróxidos.

Getoff \& Bekbölet [8] mostraram que uma solução contendo $1.0 \times 10^{-4} \mathrm{~mol}$. $\mathrm{dm}^{-3}$ de compostos clorados, aproximadamente $91 \%$ dos $\mathrm{e}^{-}{ }_{(\mathrm{aq})}$ eram convertidos de acordo com a reação (34). O restante dos $\mathrm{e}_{(\text {aq) }}^{-}$reagiam exclusivamente com o grupo aldeído da molécula [18]. Ao mesmo tempo todos átomos de $\mathrm{H}$ resultam em $\mathrm{HO}_{2}$ que posteriormente são convertidas em espécies $\mathrm{O}_{2}^{-}$(Eq. (35)). Os autores ainda relatam que o processo de degradação dos substratos é iniciado predominantemente pelos radicais $\mathrm{OH}$.

\section{Valor radiolítico- $G$}

Os G-valores encontrados para a irradiação do herbicida 2,4-D em água e em metanol confirmam mais uma vez a influência da taxa de dose na degradação., isto é os valores de $\mathrm{G}$ são maiores quando se utilizou taxa de dose maiores (5-60 kGy $\mathrm{h}^{-1}$ ). De acordo com GETOFF \& LUTZ [9], G valores nas faixas de 0,1 to $0,31 \mathrm{mmol} \mathrm{J}^{-1}$ na decomposição de compostos orgânicos indicam a ausência de reações em cadeia. Isto pode levar a formação de um número pequeno de espécies reativas requerendo assim altas dosagens para uma efetiva degradação, não sendo mais economicamente viável. Assim, os $\mathrm{G}$ valores encontrados, 0,81 and 1,04 para a degradação do 2,4-D em água indicaram a formação de grande quantidade de espécies reativas, enquanto que para o metanol, os valores encontrados, 0,14 e 0,10, indicam que neste meio, houve uma baixa formação destas espécies reativas.

\section{Conclusões}

Foi verificado através dos resultados que a dose de radiação pelo tempo de exposição (taxa de dose em $\mathrm{kGy} \mathrm{h}^{-1}$ ) teve influência significativa na degradação do herbicida 2,4-D em meio aquoso e metanólico. A degradação do herbicida foi maior quando se utilizou a taxa de dose maior (5-60 kGy $\left.\mathrm{h}^{-1}\right)$. Esses resultados são importantes para poderse entender melhor a ação da radiação gama em compostos que podem se tornar nocivos poluentes ambientais e assim, no futuro, o uso das radiações poderão se transformar em um importante método de descontaminação ambiental, principalmente na degradação de compostos que não são facilmente degradados pelos métodos mais convencionais.

\section{Agradecimentos}

Os autores agradecem a Empresa Brasileira de Radiações - EMBRARAD pela irradiação das amostras utilizadas no estudo e ao CNPq (Brasil) pelo suporte financeiro.

Recebido em: 04/08/03

Aceito em: 31/10/03

S. X. de Campos, S. M. Sanches, S. Z. Falone, E. M. Vieira. Influence of rate dose in the degradation of the 2,4 dichlorophenoxyacetic acid (2,4-D) herbicide in water and methanol by gamma radiation from cobalto- 60 .

Abstract: Conventional methods for environmental decontamination sometimes just transfer these residues from one place to another. This study deals to the study of the influence of dose rate in the degradation of 2,4-D herbicide in water and methanol solutions by gamma radiation from cobalt-60. The results showed that pure 2,4-D herbicide in water was totally degraded with a dose of radiation of $30 \mathrm{kGy}$ at a dose rate of $2,7 \mathrm{kGy} \mathrm{h}^{-1}$ and $20 \mathrm{kGy}$ with dose rates of 5- $60 \mathrm{kGy} \mathrm{h}^{-1}$. For a total degradation of pure 2,4-D herbicide in methanol to occur a dose of radiation of $150 \mathrm{kGy}$ at a dose rate of $2,7 \mathrm{kGy} \mathrm{h}^{-1}$ and $100 \mathrm{kGy}$ at a dose rate of $5-60 \mathrm{kGy} \mathrm{h}^{-1}$ were needed. It is concluded that radiolytic degradation of 2,4-D herbicide depends on of the dose and the dose rate. The radiolytic yield from 2,4-D degradation was calculated.

Keywords: 2,4-D; degradation; dose ratio; gama radiation 


\section{Referências}

[1] S. M.Branco, Natureza e agroquímicos, Moderna, São Paulo, 1991, $56 \mathrm{p}$.

[2] D. G. Hoover, G. E. Borgonovi, S. H. Jones, M. Alexander, Appl. Environ. Microb. 51 (1986) 226.

[3] S. X. Campos, E. M. Vieira, Quim. Nova. 25 (2002) 529.

[4] S. X. Campos, E. M. Vieira, J. M. P. Cordeiro, E. RodriguesFilho, M. Murgu, Phys. Chem. 2003, In press.

[5] R. B. Draper, M. A. Fox, E. Pelizzetti, N. Serpone, J. Phys. Chem. 93 (1989) 1938.

[6] X. Fang, Y. He, J. Liu, J. Wu, Radiat. Phys. Chem. 53 (1998)

411.

[7] N. Getoff, W. D. Gepper, Radiat Phys. Chem. 51 (1998)

281

[8] N. Getoff, M. Bekbölet, Radiat Phys. Chem. 56 (1999) 333.

[9] N. Getoff, W. Lutz, Radiat Phys. Chem. 25 (1985) 21.

[10] J. Hoigne, Aqueous Radiation Chemistry in Relation to
Waste Treatment. An Introductory Review, In: Radiation For Clean Environment, Proceedings International Atomic Energy Agency, Vienna ,1975, p. 297-305.

[11] R. J. Hilarides, K. A. Gray, J. Guzzetta, N. Cortellucci, C. Sommer, Environ. Sci. Technol. 28 (1994) 2234.

[12] R. J. Hilarides, K. A. Gray, Radiat. Phys. Chem. 46 (1995) 1081 .

[13] M. G. Bettoli, M. Ravanelli, L. Tositti, O. Tubertini, L. Guzzi, G. Martinotti, G. Queirazza, M. Tamba, Radiat. Phys. Chem.52 (1998) 327.

[14] P. Icre, C. Facorat, H. Rocquigny, J. C. Darbordd, Radiat. Phys. Chem. 46 (1995) 1099.

[15] J. F. Swinwood, F. M. Fraser, Radiat. Phys. Chem.46 (1995) 1147.

[16] T. Sawai, M. Sekiguchi, T. Shimokawa, T. Sawai, Radiat. Phys. Chem. 42 (1993) 723.

[17] U. Stafford, K. A. Gray, P. V. Kamat, J. Phys. Chem. 98 (1994) 6343.

[18] J. W. T. Spinks, R. J. Woods, An Introdution to Radiation Chemistry, John Wiley, New York, 1964, 477 p. 